\title{
Fixed point theorems of generalized cyclic orbital Meir-Keeler contractions
}

Chi-Ming Chen*

\section{"Correspondence:}

ming@mail.nhcue.edu.tw

Department of Applied

Mathematics, National Hsinchu

University of Education, Hsinchu,

Taiwan

\begin{abstract}
In this paper, we introduce two class of generalized cyclic orbital Meir-Keeler contractions and we study the existence and uniqueness of fixed points for these mappings. Our results in this paper extend and generalize several existing fixed-point theorems in the literature.
\end{abstract}

MSC: 47H10; 54C60; 54H25; 55M2O

Keywords: fixed-point theorem; cyclic map; generalized cyclic orbital Meir-Keeler contraction

\section{Introduction and preliminaries}

Throughout this paper, by $\mathbb{R}^{+}$, we denote the set of all non-negative numbers, while $\mathbb{N}$ is the set of all natural numbers. It is well known and easy to prove that if $(X, d)$ is a complete metric space, and if $f: X \rightarrow X$ is continuous and $f$ satisfies

$$
d\left(f x, f^{2} x\right) \leq k \cdot d(x, f x), \quad \text { for all } x \in X \text { and } k \in(0,1),
$$

then $f$ has a fixed point in $X$. Using the above conclusion, Kirk, Srinivasan and Veeramani [1] proved the following fixed-point theorem.

Theorem 1 [1] Let $A$ and $B$ be two nonempty closed subsets of a complete metric space $(X, d)$, and suppose $f: A \cup B \rightarrow A \cup B$ satisfies

(i) $f(A) \subset B$ and $f(B) \subset A$,

(ii) $d(f x, f y) \leq k \cdot d(x, y)$ for all $x \in A, y \in B$ and $k \in(0,1)$.

Then $A \cap B$ is nonempty and $f$ has a unique fixed point in $A \cap B$.

The following definitions and results will be needed in the sequel. Let $A$ and $B$ be two nonempty subsets of a metric space $(X, d)$. A mapping $f: A \cup B \rightarrow A \cup B$ is called a cyclic map if $f(A) \subseteq B$ and $f(B) \subseteq A$. In 2010, Karpagam and Agrawal [2] introduced the notion of cyclic orbital contraction, and obtained a unique fixed point theorem for such a map.

Definition 1 [2] Let $A$ and $B$ be nonempty subsets of a metric space $(X, d), f: A \cup B \rightarrow$ $A \cup B$ be a cyclic map such that for some $x \in A$, there exists a $\kappa_{x} \in(0,1)$ such that

$$
d\left(f^{2 n} x, f y\right) \leq \kappa_{x} \cdot d\left(f^{2 n-1} x, y\right), \quad n \in \mathbb{N}, y \in A .
$$

Then $f$ is called a cyclic orbital contraction.

o 2013 Chen; licensee Springer. This is an Open Access article distributed under the terms of the Creative Commons Attribution License (http://creativecommons.org/licenses/by/2.0), which permits unrestricted use, distribution, and reproduction in any medium, provided the original work is properly cited. 
Theorem 2 [2] Let $A$ and $B$ be two nonempty closed subsets of a complete metric space $(X, d)$, and let $f: A \cup B \rightarrow A \cup B$ be a cyclic orbital contraction. Then $f$ has a fixed point in $A \cap B$.

Further, many results dealing with cyclic contractions have appeared in the literature (see, e.g., [3-16]).

In 2012, Chen [17] introduced the below notion of cyclic orbital stronger Meir-Keeler contraction, and obtained a unique fixed-point theorem for such class of mappings.

Definition 2 [17] Let $(X, d)$ be a metric space. We call $\psi: \mathbb{R}^{+} \rightarrow[0,1)$ a stronger MeirKeeler type mapping in $X$ if the mapping $\psi$ satisfies the following condition:

$$
\forall \eta>0, \exists \delta>0, \exists \gamma_{\eta} \in[0,1), \forall x, y \in X \quad\left(\eta \leq d(x, y)<\delta+\eta \Rightarrow \psi(d(x, y))<\gamma_{\eta}\right) .
$$

Definition 3 [17] Let $A$ and $B$ be nonempty subsets of a metric space $(X, d)$. Suppose $f: A \cup B \rightarrow A \cup B$ is a cyclic map such that for some $x \in A$, there exists a stronger MeirKeeler type mapping $\psi_{x}: \mathbb{R}^{+} \rightarrow[0,1)$ in $X$ such that

$$
d\left(f^{2 n} x, f y\right) \leq \psi_{x}\left(d\left(f^{2 n-1} x, y\right)\right) \cdot d\left(f^{2 n-1} x, y\right)
$$

for all $n \in \mathbb{N}$ and $y \in A$. Then $f$ is called a cyclic orbital stronger Meir-Keeler $\psi_{x^{-}}$ contraction.

Clearly, if $f: A \cup B \rightarrow A \cup B$ is a cyclic orbital contraction, then $f$ is a cyclic orbital stronger Meir-Keeler $\psi_{x}$-contraction, where $\psi_{x}(t)=k_{x}$ for all $t \in \mathbb{R}^{+}$.

Theorem 3 [17] Let $A$ and $B$ be two nonempty closed subsets of a complete metric space $(X, d)$, and let $\psi_{x}: \mathbb{R}^{+} \rightarrow[0,1)$ be a stronger Meir-Keeler type mapping in $X$. Supposef $: A \cup$ $B \rightarrow A \cup B$ is a cyclic orbital stronger Meir-Keeler $\psi_{x}$-contraction. Then $A \cap B$ is nonempty and $f$ has a unique fixed point in $A \cap B$.

Chen [17] also introduced the below notion of cyclic orbital weaker Meir-Keeler contraction, and obtained a unique fixed-point theorem for such class of mappings.

Definition 4 [17] Let $(X, d)$ be a metric space, and $\psi: \mathbb{R}^{+} \rightarrow \mathbb{R}^{+}$. Then $\psi$ is called a weaker Meir-Keeler type mapping in $X$, if the mapping $\psi$ satisfies the following condition:

$$
\forall \eta>0, \exists \delta>0, \forall x, y \in X \quad\left(\eta \leq d(x, y)<\delta+\eta \Rightarrow \exists n_{0} \in \mathbb{N} \psi^{n_{0}}(d(x, y))<\eta\right) .
$$

Definition 5 [17] Let $(X, d)$ be a metric space. We call $f: \mathbb{R}^{+} \rightarrow \mathbb{R}^{+}$a $\psi$-mapping in $X$ if the function $f$ satisfies the following conditions:

$\left(\psi_{1}\right) f$ is a weaker Meir-Keeler type mapping in $X$ with $f(0)=0$;

$\left(\psi_{2}\right) \quad$ (a) if $\lim _{n \rightarrow \infty} t_{n}=\gamma>0$, then $\lim _{n \rightarrow \infty} f\left(t_{n}\right) \leq \gamma$, and

(b) if $\lim _{n \rightarrow \infty} t_{n}=0$, then $\lim _{n \rightarrow \infty} f\left(t_{n}\right)=0$;

$\left(\psi_{3}\right)\left\{f^{n}(t)\right\}_{n \in \mathbb{N}}$ is decreasing, for each $t \in \mathbb{R}^{+} \backslash\{0\}$. 
Definition 6 [17] Let $A$ and $B$ be nonempty subsets of a metric space $(X, d)$. Suppose $f: A \cup B \rightarrow A \cup B$ is a cyclic map such that for some $x \in A$, there exists a $\psi$-mapping $\psi_{x}: \mathbb{R}^{+} \rightarrow \mathbb{R}^{+}$in $X$ such that

$$
d\left(f^{2 n} x, f y\right) \leq \psi_{x}\left(d\left(f^{2 n-1} x, y\right)\right)
$$

for all $n \in \mathbb{N}$ and $y \in A$. Then $f$ is called a cyclic orbital weaker Meir-Keeler $\psi_{x}$-contraction.

Theorem 4 [17] Let $A$ and $B$ be two nonempty closed subsets of a complete metric space $(X, d)$, and let $\psi_{x}: \mathbb{R}^{+} \rightarrow \mathbb{R}^{+}$be a $\psi$-mapping in $X$. Suppose $f: A \cup B \rightarrow A \cup B$ is a cyclic orbital weaker Meir-Keeler $\psi_{x}$-contraction. Then $A \cap B$ is nonempty and $f$ has a unique fixed point in $A \cap B$.

\section{Fixed-point theorems (I)}

In this section, we will introduce the class of generalized cyclic orbital stronger MeirKeeler $\left(\psi_{x}, \varphi\right)$-contraction and we study the existence and uniqueness of fixed points for such mappings. Our results in this section extend and generalize several existing fixedpoint theorems in the literature, including Theorem 2 and Theorem 3.

In the sequel, we denote by $\Theta$ the class of functions $\varphi: \mathbb{R}^{+5} \rightarrow \mathbb{R}^{+}$satisfying the following conditions:

$\left(\varphi_{1}\right) \varphi$ is a strictly increasing, continuous function in each coordinate;

$\left(\varphi_{2}\right)$ for all $t>0, \varphi(t, t, t, 0,2 t)<t, \varphi(t, t, t, 2 t, 0)<t, \varphi(t, 0,0, t, t)<t, \varphi(0,0, t, t, 0)<t$, and $\varphi(0,0,0,0,0)=0$.

Example 1 Let $\varphi: \mathbb{R}^{+5} \rightarrow \mathbb{R}^{+}$denote

$$
\varphi\left(t_{1}, t_{2}, t_{3}, t_{4}, t_{5}\right)=\frac{2}{3} \cdot \max \left\{t_{1}, t_{2}, t_{3}, \frac{1}{2} t_{4}, \frac{1}{2} t_{5}\right\} .
$$

Then $\varphi$ satisfies the above conditions $\left(\varphi_{1}\right)$ and $\left(\varphi_{2}\right)$.

We now denote the below notion of generalized cyclic orbital stronger Meir-Keeler $\left(\psi_{x}, \varphi\right)$-contraction.

Definition 7 Let $A$ and $B$ be nonempty subsets of a metric space $(X, d)$. Suppose $f: A \cup$ $B \rightarrow A \cup B$ is a cyclic map such that for some $x \in A$, there exist a stronger Meir-Keeler type mapping $\psi_{x}: \mathbb{R}^{+} \rightarrow[0,1)$ in $X$ and $\varphi \in \Theta$ such that

$$
d\left(f^{2 n} x, f y\right) \leq \psi_{x}\left(d\left(f^{2 n-1} x, y\right)\right) \cdot \theta,
$$

where

$$
\theta=\varphi\left(d\left(f^{2 n-1} x, y\right), d\left(f^{2 n-1} x, f^{2 n} x\right), d(f y, y), d\left(f^{2 n-1} x, f y\right), d\left(f^{2 n} x, y\right)\right)
$$

for all $n \in \mathbb{N}$ and $y \in A$. Then $f$ is called a generalized cyclic orbital stronger Meir-Keeler $\left(\psi_{x}, \varphi\right)$-contraction. 
Our main result is the following.

Theorem 5 Let $A$ and $B$ be two nonempty closed subsets of a complete metric space $(X, d)$, and let $\psi_{x}: \mathbb{R}^{+} \rightarrow[0,1)$ be a stronger Meir-Keeler type mapping in $X$ and $\varphi \in \Theta$. Suppose $f: A \cup B \rightarrow A \cup B$ is a generalized cyclic orbital stronger Meir-Keeler $\left(\psi_{x}, \varphi\right)$-contraction. Then $A \cap B$ is nonempty and $f$ has a unique fixed point in $A \cap B$.

Proof Since $f: A \cup B \rightarrow A \cup B$ is a generalized cyclic orbital stronger Meir-Keeler $\left(\psi_{x}, \varphi\right)$ contraction and for $x \in A$, we have $f^{2 n} x \in A$. Put $y=f^{2 n} x$, for $n \in \mathbb{N}$. Then we have that for each $n \in \mathbb{N}$

$$
\begin{aligned}
& d\left(f^{2 n} x, f^{2 n+1} x\right) \leq \psi_{x}\left(d\left(f^{2 n-1} x, f^{2 n} x\right)\right) \cdot \theta \\
& \theta=\varphi\left(d\left(f^{2 n-1} x, f^{2 n} x\right), d\left(f^{2 n-1} x, f^{2 n} x\right), d\left(f^{2 n+1} x, f^{2 n} x\right), d\left(f^{2 n-1} x, f^{2 n+1} x\right), d\left(f^{2 n} x, f^{2 n} x\right)\right) \\
& =\varphi\left(d\left(f^{2 n-1} x, f^{2 n} x\right), d\left(f^{2 n-1} x, f^{2 n} x\right), d\left(f^{2 n+1} x, f^{2 n} x\right), d\left(f^{2 n-1} x, f^{2 n} x\right)\right. \\
& \left.\quad+d\left(f^{2 n} x, f^{2 n+1} x\right), 0\right)
\end{aligned}
$$

and by the conditions of the function $\varphi$, we get

$$
\theta<d\left(f^{2 n-1} x, f^{2 n} x\right)
$$

and

$$
\begin{aligned}
d\left(f^{2 n} x, f^{2 n+1} x\right) & <\psi_{x}\left(d\left(f^{2 n-1} x, f^{2 n} x\right)\right) \cdot d\left(f^{2 n-1} x, f^{2 n} x\right) \\
& \leq d\left(f^{2 n-1} x, f^{2 n} x\right) .
\end{aligned}
$$

Similarly, we put $y=f^{2 n} x$ and for each $n \in \mathbb{N}$

$$
\begin{aligned}
& d\left(f^{2 n+1} x, f^{2 n+2} x\right)=d\left(f^{2 n+2} x, f^{2 n+1} x\right) \\
& \leq \psi_{x}\left(d\left(f^{2 n+1} x, f^{2 n} x\right)\right) \cdot \theta \\
& \theta=\varphi\left(d\left(f^{2 n+1} x, f^{2 n} x\right), d\left(f^{2 n+1} x, f^{2 n+2} x\right), d\left(f^{2 n+1} x, f^{2 n} x\right),\right. \\
& \left.\quad d\left(f^{2 n+1} x, f^{2 n+1} x\right), d\left(f^{2 n+2} x, f^{2 n} x\right)\right) \\
& =\varphi\left(d\left(f^{2 n+1} x, f^{2 n} x\right), d\left(f^{2 n+1} x, f^{2 n+2} x\right), d\left(f^{2 n+1} x, f^{2 n} x\right), 0, d\left(f^{2 n} x, f^{2 n+1} x\right)\right. \\
& \left.\quad+d\left(f^{2 n+1} x, f^{2 n+2} x\right)\right)
\end{aligned}
$$

and by the conditions of the function $\varphi$, we get

$$
\theta<d\left(f^{2 n} x, f^{2 n+1} x\right)
$$

and

$$
\begin{aligned}
d\left(f^{2 n+1} x, f^{2 n+2} x\right) & <\psi_{x}\left(d\left(f^{2 n+1} x, f^{2 n} x\right)\right) \cdot d\left(f^{2 n} x, f^{2 n+1} x\right) \\
& \leq d\left(f^{2 n} x, f^{2 n+1} x\right) .
\end{aligned}
$$


Using inequalities (2.1) and (2.2), we deduce that $\left\{d\left(f^{n} x, f^{n+1} x\right)\right\}$ is a decreasing sequence and hence it is convergent. Let $\lim _{n \rightarrow \infty} d\left(f^{n} x, f^{n+1} x\right)=\eta$. Then there exists $\kappa_{0} \in \mathbb{N}$ and $\delta>0$ such that for all $n \geq \kappa_{0}$,

$$
\eta \leq d\left(f^{n} x, f^{n+1} x\right)<\eta+\delta .
$$

Taking into account the above inequality and the definition of stronger Meir-Keeler type mapping $\psi_{x}$ in $X$, corresponding to $\eta$ use, there exists $\gamma_{\eta} \in[0,1)$ such that

$$
\psi_{x}\left(d\left(f^{n} x, f^{n+1} x\right)\right)<\gamma_{\eta} \quad \text { for all } n \geq \kappa_{0} .
$$

Put $n_{0}=\left[\frac{\kappa_{0}+3}{2}\right]$, where $\left[\frac{\kappa_{0}+3}{2}\right]$ is the integer part of $\frac{\kappa_{0}+3}{2}$. It follows from (2.1), (2.2) and (2.3) that we deduce that for all $n \geq n_{0}$,

$$
\begin{aligned}
d\left(f^{2 n} x, f^{2 n+1} x\right) & <\psi_{x}\left(d\left(f^{2 n-1} x, f^{2 n} x\right)\right) \cdot d\left(f^{2 n-1} x, f^{2 n} x\right) \\
& <\gamma_{\eta} \cdot d\left(f^{2 n-1} x, f^{2 n} x\right),
\end{aligned}
$$

and

$$
\begin{aligned}
d\left(f^{2 n+1} x, f^{2 n+2} x\right) & <\psi_{x}\left(d\left(f^{2 n+1} x, f^{2 n} x\right)\right) \cdot d\left(f^{2 n} x, f^{2 n+1} x\right) \\
& <\gamma_{\eta} \cdot d\left(f^{2 n} x, f^{2 n+1} x\right) .
\end{aligned}
$$

It follows from (2.4) and (2.5) that for each $n \in \mathbb{N} \cup\{0\}$

$$
d\left(f^{2 n_{0}+n} x, f^{2 n_{0}+n+1} x\right)<\gamma_{\eta}^{n} \cdot d\left(f^{2 n_{0}-1} x, f^{2 n_{0}} x\right) .
$$

Since $\gamma_{\eta}<1$, we get

$$
\lim _{n \rightarrow \infty} d\left(f^{2 n_{0}+n} x, f^{2 n_{0}+n+1} x\right)=0 .
$$

For $m, n \in \mathbb{N}$ with $m>n$, we have

$$
d\left(f^{2 n_{0}+n} x, f^{2 n_{0}+m} x\right) \leq \sum_{i=n}^{m-1} d\left(f^{2 n_{0}+i} x, f^{2 n_{0}+i+1} x\right)<\frac{\gamma_{\eta}^{m-1}}{1-\gamma_{\eta}} d\left(f^{2 n_{0}} x, f^{2 n_{0}+1} x\right),
$$

and hence $d\left(f^{n} x, f^{m} x\right) \rightarrow 0$, since $0<\gamma_{\eta}<1$. So, $\left\{f^{n} x\right\}$ is a Cauchy sequence. Since $(X, d)$ is a complete metric space, $A$ and $B$ are closed, $\left\{f^{n} x\right\} \subset A \cup B$, there exists $v \in A \cup B$ such that $\lim _{n \rightarrow \infty} f^{n} x=v$. Now $\left\{f^{2 n} x\right\}$ is a sequence in $A$ and $\left\{f^{2 n+1} x\right\}$ is a sequence in $B$, and also both converge to $v$. Since $A$ and $B$ are closed, $v \in A \cap B$, and so $A \cap B$ is nonempty. Next, we want to show that $v$ is a fixed point of $f$. Suppose that $v$ is not a fixed point of $f$. Then $d(v, f v)>0$. Since $\lim _{n \rightarrow \infty} d\left(f^{2 n-1} x, v\right)=0$ and

$$
d\left(f^{2 n} x, f v\right) \leq \psi_{x}\left(d\left(f^{2 n-1} x, v\right)\right) \cdot \theta,
$$

where

$$
\theta=\varphi\left(d\left(f^{2 n-1} x, v\right), d\left(f^{2 n-1} x, f^{2 n} x\right), d(f v, v), d\left(f^{2 n-1} x, f v\right), d\left(f^{2 n} x, v\right)\right),
$$


we obtain that

$$
\begin{aligned}
d(v, f v) & =\lim _{n \rightarrow \infty} d\left(f^{2 n} x, f v\right) \\
& \leq \gamma_{\eta} \cdot \varphi(d(v, v), d(v, v), d(f v, v), d(v, f v), d(v, v)) \\
& \leq \varphi(0,0, d(v, f v), d(v, f v), 0) \\
& <d(v, f v) .
\end{aligned}
$$

This leads to a contradiction. So, $d(v, f v)=0$, that is, $v$ is a fixed point of $f$.

Finally, we want to show the uniqueness of the fixed point. Let $\mu$ be another fixed point of $f$. By the cyclic character of $f$, we have $v, \mu \in A \cap B$. Since $f$ is a generalized cyclic orbital stronger Meir-Keeler $\left(\psi_{x}, \varphi\right)$-contraction, we have

$$
d(\nu, \mu)=d(\nu, f \mu)=\lim _{n \rightarrow \infty} d\left(f^{2 n} x, f \mu\right),
$$

and

$$
d\left(f^{2 n} x, f \mu\right) \leq \psi_{x}\left(d\left(f^{2 n-1} x, \mu\right)\right) \cdot \theta<\gamma_{\eta} \cdot \theta,
$$

where

$$
\theta=\varphi\left(d\left(f^{2 n-1} x, \mu\right), d\left(f^{2 n-1} x, f^{2 n} x\right), d(f \mu, \mu), d\left(f^{2 n-1} x, f \mu\right), d\left(f^{2 n} x, \mu\right)\right) .
$$

It follows from (2.7), (2.8) and the condition $\left(\varphi_{2}\right)$ of the mapping $\varphi$ that

$$
\begin{aligned}
d(\nu, \mu) & <\gamma_{\eta} \cdot \varphi(d(v, \mu), d(v, v), d(f \mu, \mu), d(\nu, f \mu), d(\nu, \mu)) \\
& \leq \varphi(d(v, \mu), 0,0, d(v, \mu), d(\nu, \mu)) \\
& <d(\nu, \mu) .
\end{aligned}
$$

This leads to a contradiction. Therefore, $v=\mu$, and so $v$ is the unique fixed point of $f$.

We give the following example to illustrate Theorem 5 .

Example 2 Let $A=B=X=\mathbb{R}^{+}$and we define $d: X \times X \rightarrow \mathbb{R}^{+}$by

$$
d(x, y)=|x-y|, \quad \text { for } x, y \in X,
$$

and let $f: X \rightarrow X$ denote

$$
f(x)= \begin{cases}0, & \text { if } 0 \leq x<1 \\ \frac{1}{16}, & \text { if } x \geq 1\end{cases}
$$

We next define $\psi_{x}: \mathbb{R}^{+} \rightarrow[0,1)$ by

$$
\psi_{x}(t)= \begin{cases}\frac{1}{3}, & \text { if } 0 \leq t \leq 1 \\ \frac{t}{t+1}, & \text { if } t>1\end{cases}
$$


and let $\varphi: \mathbb{R}^{+5} \rightarrow \mathbb{R}^{+}$denote

$$
\varphi\left(t_{1}, t_{2}, t_{3}, t_{4}, t_{5}\right)=\frac{1}{2} \cdot \max \left\{t_{1}, t_{2}, t_{3}, \frac{1}{2} t_{4}, \frac{1}{2} t_{5}\right\} .
$$

Then $f$ is a generalized cyclic orbital stronger Meir-Keeler $\left(\psi_{x}, \varphi\right)$-contraction and 0 is the unique fixed point.

\section{Fixed-point theorems (II)}

In this section, we will introduce the class of generalized cyclic orbital weaker Meir-Keeler $\left(\psi_{x}, \phi\right)$-contraction and we study the existence and uniqueness of fixed points for such mappings.

In the sequel, we denote by $\Phi$ the class of functions $\phi: \mathbb{R}^{+} \rightarrow \mathbb{R}^{+}$satisfying the following conditions:

$\left(\phi_{1}\right) \phi$ is lower semi-continuous, and

$\left(\phi_{2}\right) \phi(0)=0$ if and only if $t=0$.

Definition 8 Let $A$ and $B$ be nonempty subsets of a metric space $(X, d)$. Suppose $f: A \cup$ $B \rightarrow A \cup B$ is a cyclic map such that for some $x \in A$, there exist a $\psi$-mapping $\psi_{x}: \mathbb{R}^{+} \rightarrow \mathbb{R}^{+}$ in $X$ and $\phi \in \Phi$ such that

$$
d\left(f^{2 n} x, f y\right) \leq \psi_{x}\left(d\left(f^{2 n-1} x, y\right)\right)-\phi\left(d\left(f^{2 n-1} x, y\right)\right), \quad n \in \mathbb{N}, y \in A
$$

Then $f$ is called a generalized cyclic orbital weaker Meir-Keeler $\left(\psi_{x}, \phi\right)$-contraction.

Our second main result is the following.

Theorem 6 Let $A$ and $B$ be two nonempty closed subsets of a complete metric space $(X, d)$, and let $\psi_{x}: \mathbb{R}^{+} \rightarrow \mathbb{R}^{+}$be a $\psi$-mapping in $X$ and $\phi \in \Phi$. Suppose $f: A \cup B \rightarrow A \cup B$ is a generalized cyclic orbital weaker Meir-Keeler $\left(\psi_{x}, \phi\right)$-contraction. Then $A \cap B$ is nonempty and $f$ has a unique fixed point in $A \cap B$.

Proof Since $f: A \cup B \rightarrow A \cup B$ is a generalized cyclic orbital weaker Meir-Keeler $\left(\psi_{x}, \phi\right)$ contraction and for $x \in X$, there exist a $\psi$-mapping $\psi_{x}: \mathbb{R}^{+} \rightarrow \mathbb{R}^{+}$in $X$ and $\phi \in \Phi$ such that (3.1) is satisfied. Put $y=f^{2 n} x$ for all $n \in \mathbb{N}$. Then we have that for each $n \in \mathbb{N}$

$$
\begin{aligned}
d\left(f^{2 n} x, f^{2 n+1} x\right) & \leq \psi_{x}\left(d\left(f^{2 n-1} x, f^{2 n} x\right)\right)-\phi\left(d\left(f^{2 n-1} x, f^{2 n} x\right)\right) \\
& \leq \psi_{x}\left(d\left(f^{2 n-1} x, f^{2 n} x\right)\right)
\end{aligned}
$$

and

$$
\begin{aligned}
d\left(f^{2 n+1} x, f^{2 n+2} x\right) & =d\left(f^{2 n+2} x, f^{2 n+1} x\right) \\
& \leq \psi_{x}\left(d\left(f^{2 n+1} x, f^{2 n} x\right)\right)-\phi\left(d\left(f^{2 n+1} x, f^{2 n} x\right)\right) \\
& \leq \psi_{x}\left(d\left(f^{2 n+1} x, f^{2 n} x\right)\right) .
\end{aligned}
$$


Generally, we have that for each $n \in \mathbb{N}$

$$
d\left(f^{n} x, f^{n+1} x\right) \leq \psi_{x}\left(d\left(f^{n-1} x, f^{n} x\right)\right)
$$

and so we conclude that for each $n \in \mathbb{N}$

$$
\begin{aligned}
d\left(f^{n} x, f^{n+1} x\right) & \leq \psi_{x}\left(d\left(f^{n-1} x, f^{n} x\right)\right) \\
& \leq \psi_{x}^{2}\left(d\left(f^{n-2} x, f^{n-1} x\right)\right) \\
& \leq \cdots \\
& \leq \psi_{x}^{n}(d(x, f x)) .
\end{aligned}
$$

Since $\left\{\psi_{x}^{n}(d(x, f x))\right\}_{n \in \mathbb{N}}$ is decreasing, it must converge to some $\eta \geq 0$. We claim that $\eta=0$. On the contrary, assume that $\eta>0$. Then by the definition of weaker Meir-Keeler type mapping $\psi_{x}$ in $X$, there exists $\delta>0$ such that for $x, y \in X$ with $\eta \leq d(x, y)<\delta+\eta$, there exists $n_{0} \in \mathbb{N}$ such that $\psi_{x}^{n_{0}}(d(x, y))<\eta$. Since $\lim _{n \rightarrow \infty} \psi_{x}^{n}(d(x, f x))=\eta$, there exists $m_{0} \in \mathbb{N}$ such that $\eta \leq \psi_{x}^{m}(d(x, f x))<\delta+\eta$, for all $m \geq m_{0}$. Thus, we conclude that $\psi_{x}^{m_{0}+n_{0}}\left(d\left(x_{0}, x_{1}\right)\right)<\eta$, and we get a contradiction. So, $\lim _{n \rightarrow \infty} \psi_{x}^{n}(d(x, f x))=0$, that is,

$$
\lim _{n \rightarrow \infty} d\left(f^{n} x, f^{n+1} x\right)=0
$$

We now claim that $\left\{f^{n} x\right\}$ is a Cauchy sequence. It is sufficient to show that $\left\{f^{2 n} x\right\}$ is a Cauchy sequence. Suppose $\left\{f^{2 n} x\right\}$ is not Cauchy. Then there exists $\varepsilon>0$ such that for all $k \in \mathbb{N}$, there are $m_{k}, n_{k} \in \mathbb{N}$ with $m_{k}>n_{k} \geq k$ satisfying:

(i) $d\left(f^{2 m_{k}} x, f^{2 n_{k}}\right) \geq \varepsilon$, and

(ii) $m_{k}$ is the smallest number greater than $n_{k}$ such that the condition (i) holds.

Using (3.2), we have

$$
\begin{aligned}
\varepsilon & \leq d\left(f^{2 m_{k}} x, f^{2 n_{k}}\right) \leq d\left(f^{2 m_{k}} x, f^{2 m_{k}-1}\right)+d\left(f^{2 m_{k}-1} x, f^{2 m_{k}-2}\right)+d\left(f^{2 m_{k}-2} x, f^{2 n_{k}}\right) \\
& \leq d\left(f^{2 m_{k}} x, f^{2 m_{k}-1}\right)+d\left(f^{2 m_{k}-1} x, f^{2 m_{k}-2}\right)+\varepsilon .
\end{aligned}
$$

Let $k \rightarrow \infty$, we get

$$
\lim _{n \rightarrow \infty} d\left(f^{2 m_{k}} x, f^{2 n_{k}}\right)=\varepsilon
$$

On the other hand, applying (3.1) with $y=f^{2 n_{k}} x$ for all $k \in \mathbb{N}$, we get

$$
d\left(f^{2 m_{k}} x, f^{2 n_{k}+1}\right) \leq \psi_{x}\left(d\left(f^{2 m_{k}-1} x, f^{2 n_{k}}\right)\right)-\phi\left(d\left(f^{2 m_{k}-1} x, f^{2 n_{k}}\right)\right) .
$$

Since for each $k \in \mathbb{N}$

$$
d\left(f^{2 m_{k}} x, f^{2 n_{k}+1}\right) \leq d\left(f^{2 m_{k}} x, f^{2 n_{k}}\right)+d\left(f^{2 n_{k}} x, f^{2 n_{k}+1}\right),
$$

and

$$
d\left(f^{2 m_{k}-1} x, f^{2 n_{k}}\right) \leq d\left(f^{2 m_{k}-1} x, f^{2 m_{k}}\right)+d\left(f^{2 m_{k}} x, f^{2 n_{k}}\right),
$$


taking $k \rightarrow \infty$ and using the inequalities (3.3), (3.5) and (3.6), we get

$$
\lim _{n \rightarrow \infty} d\left(f^{2 m_{k}} x, f^{2 n_{k}+1}\right)=\varepsilon,
$$

and

$$
\lim _{n \rightarrow \infty} d\left(f^{2 m_{k}-1} x, f^{2 n_{k}}\right)=\varepsilon
$$

Taking into account the inequalities (3.4), (3.7) and (3.8), and by the definitions of the functions $\phi$ and $\psi_{x}$, we get

$$
\begin{aligned}
\varepsilon & =\lim _{n \rightarrow \infty} d\left(f^{2 m_{k}} x, f^{2 n_{k}+1}\right) \\
& \leq \lim _{n \rightarrow \infty} \psi_{x}\left(d\left(f^{2 m_{k}-1} x, f^{2 n_{k}}\right)\right)-\lim _{n \rightarrow \infty} \phi\left(d\left(f^{2 m_{k}-1} x, f^{2 n_{k}}\right)\right) \\
& \leq \varepsilon-\phi(\varepsilon),
\end{aligned}
$$

which implies that $\varepsilon=0$. Thus, $\left\{f^{n} x\right\}$ is a Cauchy sequence.

Since $(X, d)$ is a complete metric space, $A$ and $B$ are closed, $\left\{f^{n} x\right\} \subset A \cup B$, there exists $v \in$ $A \cup B$ such that $\lim _{n \rightarrow \infty} f^{n} x=v$. Now $\left\{f^{2 n} x\right\}$ is a sequence in $A$ and $\left\{f^{2 n+1} x\right\}$ is a sequence in $B$, and also both converge to $v$. Since $A$ and $B$ are closed, $v \in A \cap B$, and so $A \cap B$ is nonempty. On the other hand, since $\lim _{n \rightarrow \infty} d\left(f^{2 n-1} x, v\right)=0$ and

$$
d\left(f^{2 n} x, f v\right) \leq \psi_{x}\left(d\left(f^{2 n-1} x, v\right)\right)-\phi\left(d\left(f^{2 n-1} x, v\right)\right),
$$

taking $n \rightarrow \infty$,we obtain that

$$
d(v, f v) \leq 0-\phi(d(v, v))=0
$$

and hence $d(v, f v)=0$, that is, $v$ is a fixed point of $f$.

Finally, we want to show the uniqueness of the fixed point. Let $\mu$ be another fixed point of $f$. By the cyclic character of $f$, we have $v, \mu \in A \cap B$. Since $f$ is a generalized cyclic orbital weaker Meir-Keeler $\left(\psi_{x}, \phi\right)$-contraction, we have

$$
d\left(f^{2 n} x, f \mu\right) \leq \psi_{x}\left(d\left(f^{2 n-1} x, \mu\right)\right)-\phi\left(d\left(f^{2 n-1} x, \mu\right)\right) .
$$

Letting $n \rightarrow \infty$, and by the definitions of the functions $\phi$ and $\psi_{x}$, we obtain that

$$
d(v, \mu)=d(v, f \mu)=\lim _{n \rightarrow \infty} d\left(f^{2 n} x, f \mu\right) \leq d(v, \mu)-\phi(d(v, \mu)),
$$

which implies that $d(v, \mu)=0$. Therefore, $v=\mu$, and so $v$ is the unique fixed point of $f$.

We give the following example to illustrate Theorem 6 .

Example 3 Let $A=B=X=\mathbb{R}^{+}$and we define $d: X \times X \rightarrow \mathbb{R}^{+}$by

$$
d(x, y)=|x-y|, \quad \text { for } x, y \in X .
$$


Define $f: X \rightarrow X$ by

$$
f(x)= \begin{cases}0, & \text { if } 0 \leq x<1 \\ \frac{1}{16}, & \text { if } x \geq 1\end{cases}
$$

and define $\psi, \phi: \mathbb{R}^{+} \rightarrow \mathbb{R}^{+}$by

$$
\psi_{x}(t)=\frac{1}{3} t \quad \text { and } \quad \phi(t)=\frac{1}{6} t \quad \text { for } t \in \mathbb{R}^{+}
$$

Then $f$ is a generalized cyclic orbital weaker Meir-Keeler $\left(\psi_{x}, \phi\right)$-contraction and 0 is the unique fixed point.

\section{Competing interests}

The author declares that they have no competing interests.

\section{Acknowledgements}

This research was supported by the National Science Council of the Republic of China. The authors would like to thank referee(s) for many useful comments and suggestions for the improvement of the paper.

\section{Received: 30 October 2012 Accepted: 24 March 2013 Published: 10 April 2013}

\section{References}

1. Kirk, WA, Srinivasan, PS, Veeramani, P: Fixed points for mappings satisfying cyclical contractive conditions. Fixed Point Theory 4(1), 79-89 (2003)

2. Karpagam, S, Agrawal, S: Best proximity point theorems for cyclic orbital Meir-Keeler contraction maps. Nonlinear Anal. 74, 1040-1046 (2010)

3. Chen, $\mathrm{CM}$, Chen, $\mathrm{CH}$ : Best proximity point theorems for some new cyclic mappings. J. Appl. Math. 2012, Article ID 643729 (2012). doi:10.1155/2012/643729

4. Chen, CM, Lin, CJ: Best periodic proximity points for cyclic weaker Meir-Keeler contractions. J. Appl. Math. 2012 Article ID 782389 (2012). doi:10.1155/2012/782389

5. Chen, CM: Fixed point theory for the cyclic weaker Meir-Keeler function in complete metric spaces. Fixed Point Theory Appl. 2012, Article ID 17 (2012)

6. Karpagam, S, Agrawal, S: Existence of best proximity points of $p$-cyclic contractions. Fixed Point Theory 13(1), 99-105 (2012)

7. Karapinar, E: Fixed point theory for cyclic weak $\phi$-contraction. Appl. Math. Lett. 24, 822-825 (2011)

8. Karapinar, E: Best proximity points of cyclic mappings. Appl. Math. Lett. 25, 1761-1766 (2012)

9. Karapinar, E, Erhan, IM, Ulus, AY: Fixed point theorem for cyclic maps on partial metric spaces. Appl. Math. Inf. Sci. 6(1), 239-244 (2012)

10. Karapinar, E, Sadarangani, K: Fixed point theory for cyclic $(\psi-\phi)$-contractions. Fixed Point Theory Appl. 2011, Article ID 69 (2011)

11. Karapinar, E, Erhan, IM: Cyclic contractions and fixed point theorems. Filomat 26(4), 777-782 (2012)

12. Karapinar, E, Petrusel, G, Tas, K: Best proximity point theorems for $K T$-types cyclic orbital contraction mappings. Fixed Point Theory 13(2), 537-546 (2012)

13. Karapinar, E, Jleli, M, Samet, B: Fixed point results for almost generalized cyclic $(\psi, \varphi)$-weak contractive type mappings with applications. Abstr. Appl. Anal. 2012, Article ID 917831 (2012)

14. Karapinar, E, Romaguera, S, Tas, K: Fixed points for cyclic orbital generalized contractions on complete metric spaces. Cent. Eur. J. Math. 11(3), 552-560 (2013)

15. Pǎcurar, M, Rus, IA: Fixed point theory for cyclic $\varphi$-contractions. Nonlinear Anal. 72(3-4), 1181-1187 (2010)

16. Rezapour, S, Derafshpour, M, Shahzad, N: Best proximity point of cyclic $\varphi$-contractions in ordered metric spaces. Topol. Methods Nonlinear Anal. 37(1), 193-202 (2011)

17. Chen, CM: Fixed point theorems for cyclic Meir-Keeler type mappings in complete metric spaces. Fixed Point Theory Appl. 2012, Article ID 41 (2012)

doi:10.1186/1687-1812-2013-91

Cite this article as: Chen: Fixed point theorems of generalized cyclic orbital Meir-Keeler contractions. Fixed Point

Theory and Applications 2013 2013:91. 